\title{
Energy Band Structure and its Characteristics of a Two-Dimensional Metallic Photonic Crystal
}

\author{
Xianfeng $\mathrm{Bao}^{\mathrm{a}}$ and Duanzheng $\mathrm{Yao}^{\mathrm{a}, \mathrm{b}}$ \\ ${ }^{a}$ Department of Physics, Wuhan University, Wuhan 430072, China \\ ${ }^{\mathrm{b}}$ Key Laboratory of Acoustic and Photonics Material and Devices, Ministry of Education, Wuhan \\ University, Wuhan 430072, China \\ Reprint requests to D. Y.; Fax: 8627 68752569; E-mail: dzyao@whu.edu.cn \\ Z. Naturforsch. 66a, 339 -344 (2011); received September 28, 2010 / revised November 30, 2010
}

The energy band structure and its characteristics of a two-dimensional metallic photonic crystal with square lattice structure have been studied by using the finite-difference time-domain (FDTD) algorithm. In order to determine the band structure accurately, the spatial distribution of the eigenfunction has been analyzed. By comparing the distribution of different wave vectors and of different energy band eigenmodes, an effective method to determine the band structure was found, which has been verified by the simulation results.

Key words: Metallic Photonic Crystal; FDTD; Eigenmode; Band Structure.

\section{Introduction}

Photonic crystals [1 -3$]$ are a new type of functional materials with artificial structure. Due to its periodic structure, the photonic crystal shows the characteristics of the photonic band gap and the photon location and has broad applications. Especially the metallic photonic crystals, used in many fields in recent years [4,5], show many advantages. Therefore it is very important and meaningful to study the properties of the band structure of the metallic photonic crystals.

The finite-difference time-domain (FDTD) method is an effective tool for studying the band structure and its characteristics of the photonic crystals. Raineri et al. [6] presented a simple 2D FDTD code able to describe the second-harmonic generation in $1 \mathrm{D}$ or $2 \mathrm{D}$ structured materials, which is applied to a semiconductor defective photonic crystal waveguide. Zheng [7] studied the wave propagation in optical wave-guide devices. It is shown from the researches that the FDTD method is very effective for studying photonic crystals. One of the important features of this method is that it can be used to study the absorption and the dispersion of the photonic crystals.

In this paper, we used the Drude model [8] to deal with the metal dielectric, and the FDTD method has been used to simulate a 2-D metallic (gold) photonic crystal with square lattice structure. An effective method to determine the band structure was found, that is, the eigenfunctions vary widely with the same wave vectors and slowly with the same energy bands.

\section{Model and Theory}

The schematic of a two-dimensional metal photonic crystal with square lattice in the $x, y$-plane is shown in Figure 1. The lattice constant $a$ is $0.6 \mathrm{~mm}$. The cross sectional radius $r$ of the gold rods is fixed at $0.2 a$. The dielectric constant $\varepsilon_{\mathrm{b}}(\mathbf{r})$ of the medium around the rods is 1.0. We divided the unit cell into $41 \times 41$ discretized grid points. The computational grid point coordinates are given in Figure $1 \mathrm{~b}$ and only the transmission (TM) polarization is considered in the paper.

The FDTD algorithm is based on the difference of the Maxwell's equations,

$$
\begin{aligned}
& \nabla \times \mathbf{E}=-\mu_{0} \frac{\partial \mathbf{H}}{\partial t}, \\
& \nabla \times \mathbf{H}=\frac{\partial \mathbf{D}}{\partial t},
\end{aligned}
$$

where D stands for the electric displacement vector and is given by the convolution integral of the electric field $\mathbf{E}$ and the dielectric response function $\Phi$,

$$
\mathbf{D}(t)=\varepsilon_{0} \int_{-\infty}^{\infty} \mathrm{d} t^{\prime} \Phi\left(t-t^{\prime}\right) \mathbf{E}\left(t^{\prime}\right),
$$




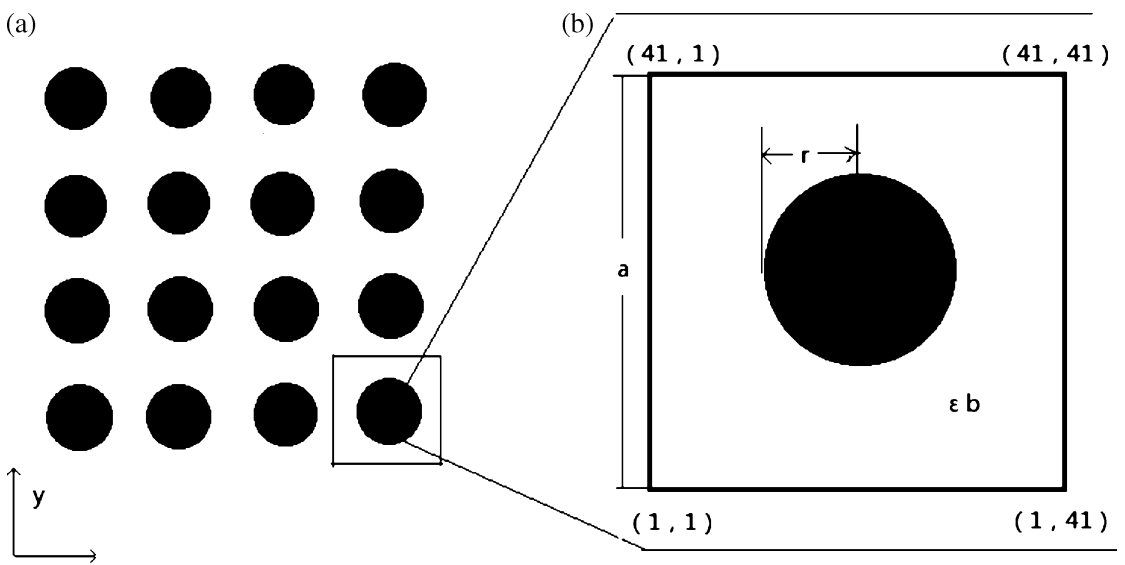

$x$

Fig. 1. Schematic of a 2D metal photonic crystal with the square lattice in the $x, y$-plane.

where $\Phi$ is the Fourier transform of the dielectric constant $\varepsilon_{\mathrm{r}}(\omega)$.

$$
\Phi(t)=\frac{1}{2 \pi} \int_{-\infty}^{+\infty} \varepsilon_{\mathrm{r}}(\omega) \exp (-\mathrm{i} \omega t) \mathrm{d} \omega .
$$

The dielectric constant for the metal is described by the Drude model,

$$
\varepsilon_{\mathrm{r}}(\omega)=\varepsilon_{\infty}\left[1-\frac{\omega_{p}^{2}}{\omega^{2}+\mathrm{i} \omega \gamma}\right],
$$

where $\varepsilon_{\infty}$ is the dielectric constant for the metal at sufficiently high frequencies, $\omega_{\mathrm{p}}$ the plasma frequency, and $\gamma$ the relaxation rate in the Drude model.

The relationship between $\mathbf{D}$ and $\mathbf{E}$ in the metal region has been obtained from (3), (4), and (5) to

$$
\begin{aligned}
\frac{1}{\varepsilon_{0}} \frac{\partial \mathbf{D}(\mathbf{r}, t)}{\partial t}= & \varepsilon_{\infty} \frac{\partial \mathbf{E}(\mathbf{r}, t)}{\partial t} \\
& +\varepsilon_{\infty} \omega_{\mathrm{p}}^{2} \int_{0}^{+\infty} \mathrm{d} t^{\prime} \exp (-\gamma t) \mathbf{E}\left(\mathbf{r}, t-t^{\prime}\right) .
\end{aligned}
$$

The relationship between $\mathbf{D}$ and $\mathbf{E}$ in the non-metallic region is very simple and reads

$$
\mathbf{D}(\mathbf{r}, t)=\varepsilon_{0} \varepsilon_{\mathrm{b}}(\mathbf{r}) \mathbf{E}(\mathbf{r}, t)
$$

Since then, we can make (1) and (2) discrete by the Yee method in the different regions [9]. It is worth notice that the FDTD method is the simulation of eletromagnetic problems in a limited space, therefore we need recommend boundary conditions:

$$
\begin{aligned}
\vec{E}(\vec{r}+\vec{a}, \mathrm{t}) & =\exp (\mathrm{i} \vec{k} \cdot \vec{a}) \vec{E}(\vec{r}, \mathrm{t}), \\
\vec{H}(\vec{r}+\vec{a}, \mathrm{t}) & =\exp (\mathrm{i} \vec{k} \cdot \vec{a}) \vec{H}(\vec{r}, \mathrm{t}),
\end{aligned}
$$

where $\vec{k}$ is the wave vector in the first Brillouin zone and $\vec{a}$ the elementary lattice vector. The resonance frequency as a function of $\vec{k}$ has been calculated in order to get the energy band structure.

The FDTD simulated time series is the linear superpostion of all eigenfunctions. In order to obtain the eigenfunction of a single eigenfrequency, we have to do a separation. The Bruch theorems can be expressed as:

$$
\begin{aligned}
& \vec{E}(r)=\exp (\mathrm{i} \vec{k} \cdot \vec{r}-\mathrm{i} \omega \mathrm{t}) \vec{u}(r), \\
& \vec{u}(r)=\exp (-\mathrm{i} \vec{k} \cdot \vec{r}+\mathrm{i} \omega \mathrm{t}) \vec{E}(r),
\end{aligned}
$$

where $\vec{u}(r)$ corresponds to the eigenfunction.

Using trigonometic orthogonality, we obtain

$$
\begin{gathered}
\sum_{n=1}^{n_{\text {max }}} \exp \left(\mathrm{i} \omega_{j} n \Delta t\right) \exp \left(-\mathrm{i} \omega_{k} n \Delta t\right) \\
= \begin{cases}n_{\max } & (j=k), \\
0 & (j \neq k),\end{cases}
\end{gathered}
$$

and the eigenfunction of a single eigenfrequency:

$$
\begin{aligned}
u\left(r, \omega_{j}\right)= & \frac{1}{n_{\max }} \exp (-\mathrm{i} \vec{k} \cdot \vec{r}) \\
& \cdot \sum_{n=1}^{n_{\max }} \Psi(r, n) \exp \left(\mathrm{i} \omega_{j} n \Delta t\right),
\end{aligned}
$$


(a)

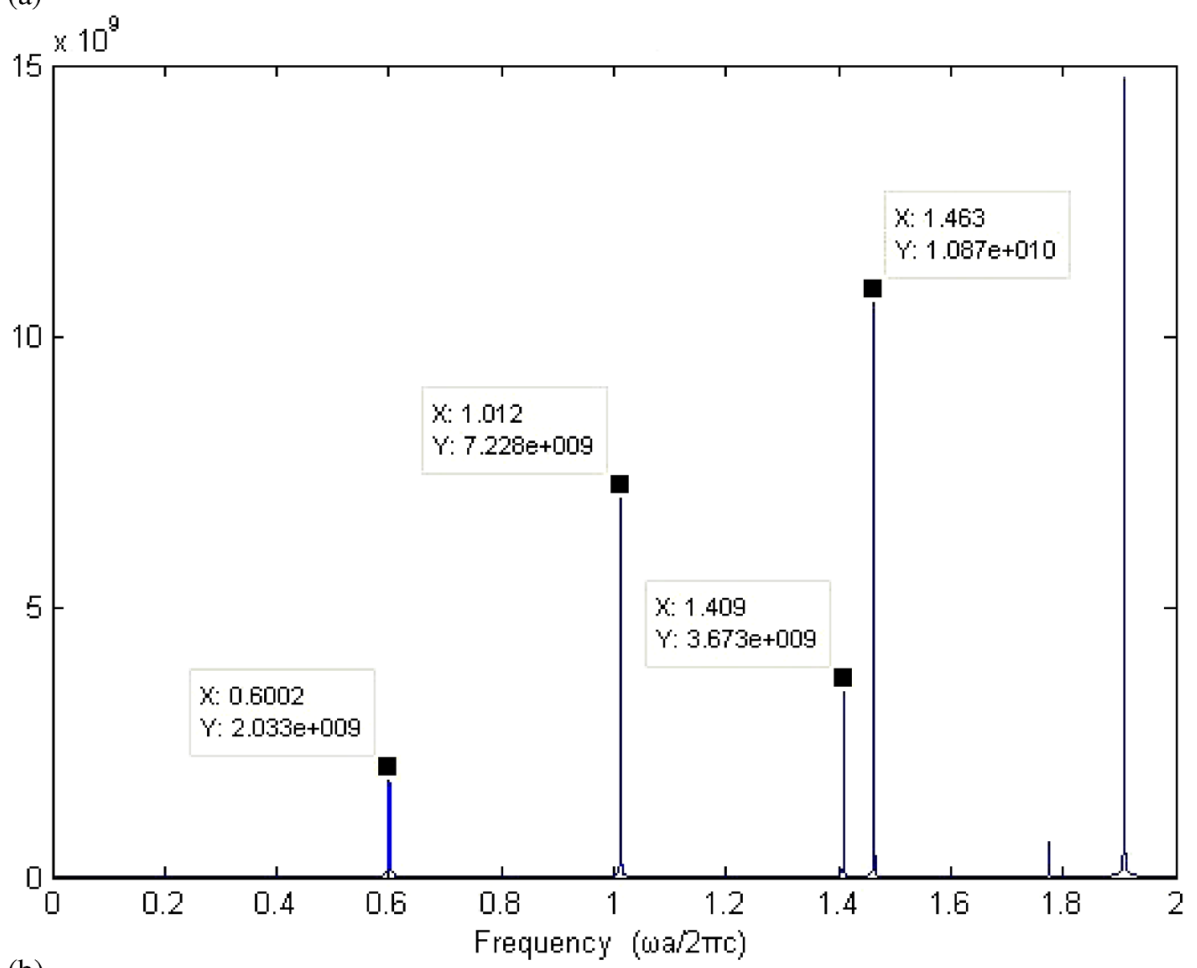

(b)

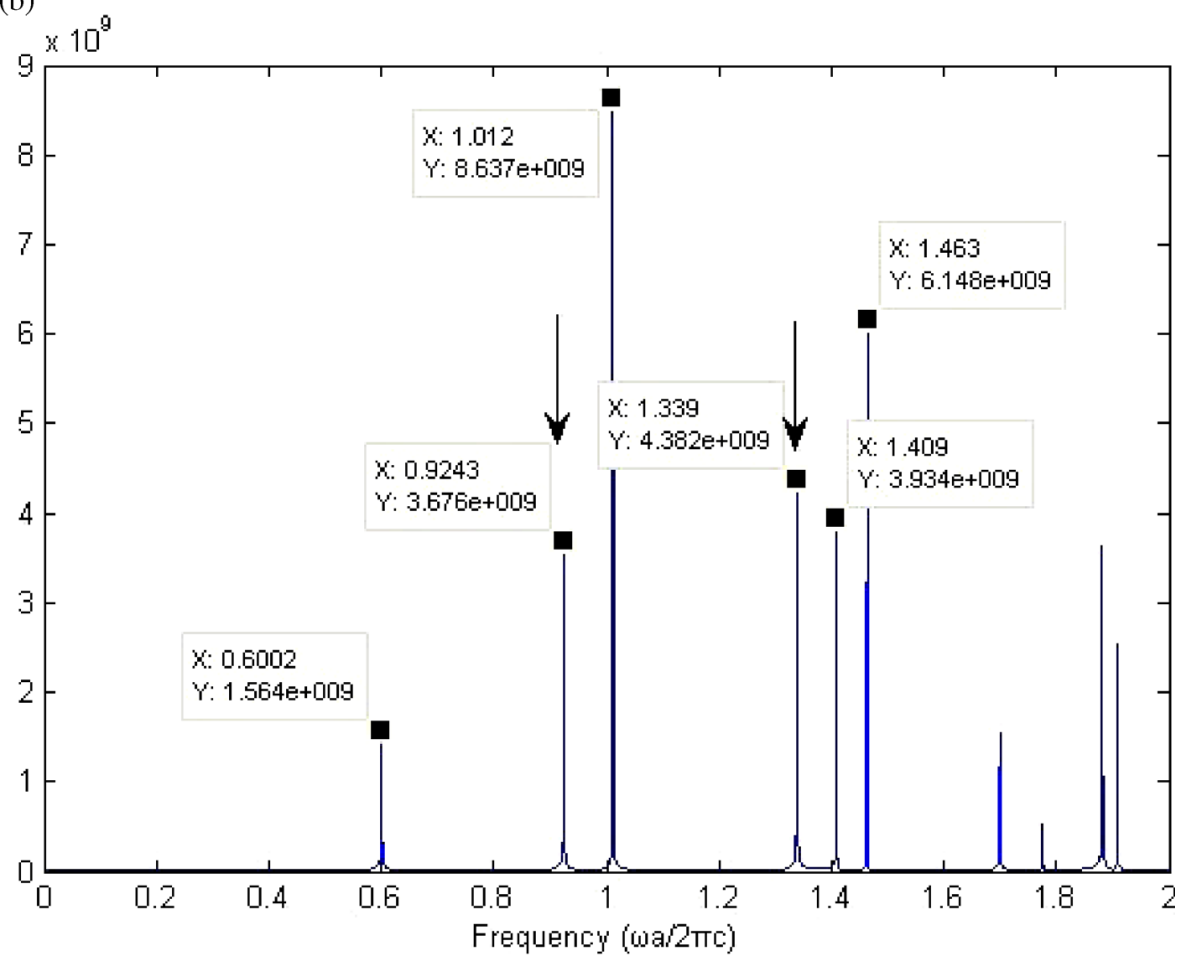

Fig. 2 (colour online). (a) Eigenspectrum of grid point $(03,03)$, where $k_{x}=k_{y}=0.75$; (b) Eigenspectrum of grid point $(37,30)$, where $k_{x}=k_{y}=0.75$. 


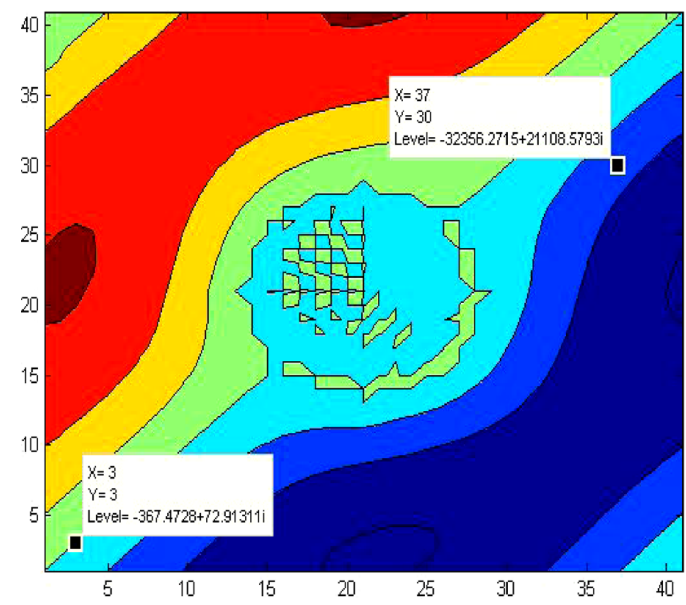

Fig. 3 (colour online). Distribution of the electric field of the eigenmode, where the eigenfrequency is 0.9243 .

(a)

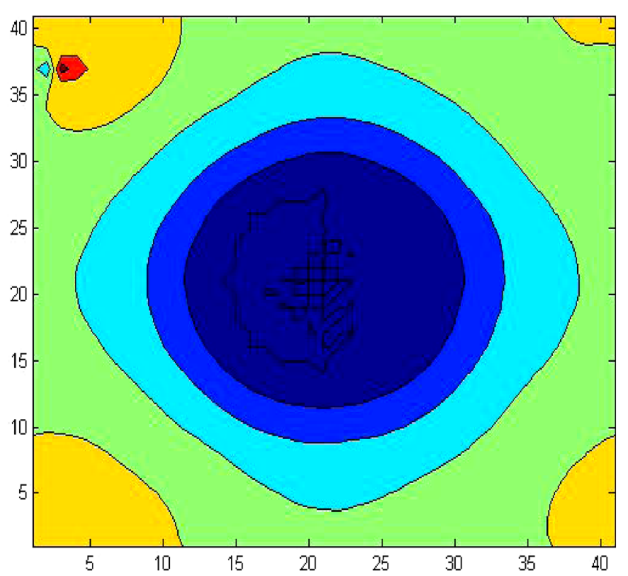

(c)

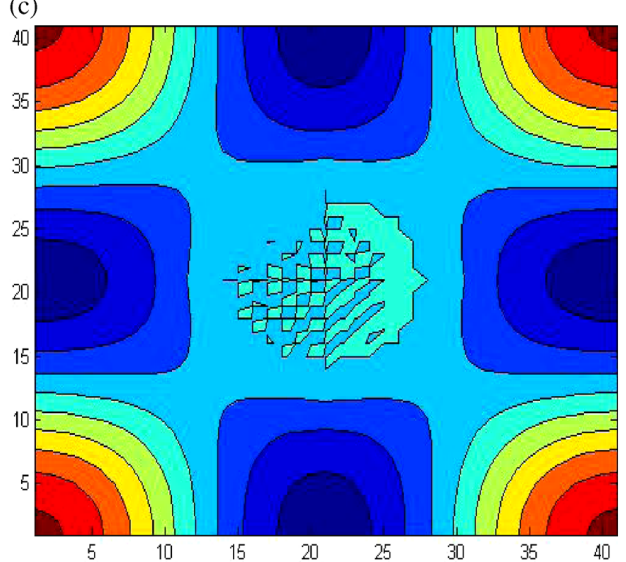

(b) where $\Psi(r, n)$ is the FDTD simulated time series in $\mathbf{r}, \omega_{j}$ the eigenfrequency of the wave vector $\vec{k}, \Delta t$ the interval of time, $n$ the time step, $n_{\max }$ is the maximum of $n$.

\section{Calculation, Results, and Analysis}

The output of the laser used in the calculation is a Gaussian beam. For a given wave vector $\mathbf{k}=$ $\left(k_{x}, k_{y}\right) \pi / a$, in order to obtain the resonance frequency (eigenfrequency), it is necessary to do a Fast fourier transform (FFT) to the FDTD simulated time series of one grid point. If the grid point selected is not very suitable, some eigenmodes may be missing. So it is difficult to distinguish the band informations of eigenfrequencies accurately.
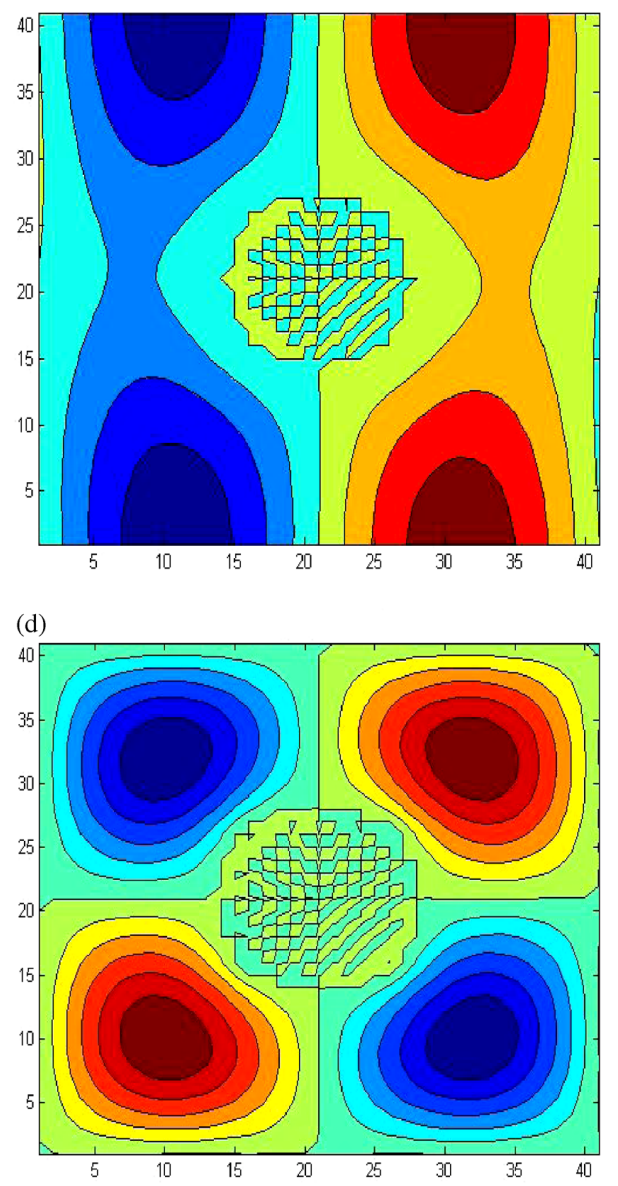

Fig. 4 (colour online). Distribution of the electric field of the eigenmode at different energy bands with the same wave vector $\left(k_{x}=k_{y}=0\right)$. (a) at the first energy band; (b) at the second energy band; (c) at the third energy band; (d) at the fourth energy band. 
The FFT results of two different grid points are shown in Figure 2: (a) the eigenspectra of grid point $(03,03)$ and (b) the eigenspectra of grid point $(37,30)$. The horizontal axis shows the normalized frequency and the vertical one the amplitude which depends on the amplitude of the Gaussian pulse and the frequency. Comparing (a) and (b), we find that the eigenmodes of the normalized frequencies 0.9243 and 1.4630 are missing in (a).

The distribution of the electric field of the eigenmode is shown in Figure 3, where the eigenfrequency $\omega$ is 0.9243 . On the grid point $(37,30)$, the electric field strength is $-32256+21108 \mathrm{i}$, the electric field strength of grid point $(03,03)$ is just $-367+72 \mathrm{i}$. It can be seen from the results that the corresponding eigenmode may be missing, when the grid point's electric field of the eigenmode is weak. It provided some sug- gestions to select the appropriate grid point and avoiding to select the grid point in the metal region.

Figure 4 shows the contour charts of eigenfunction at different energy bands with the same wave vector. The results shown in (a), (b), (c), and (d) are very different to the distribution of the electric field. It has been known from linear algebra that the eigenfunctions of different eigenmodes are linearly independent, so their spatial distribution must vary widely and differently. The results, shown in Figure 4, confirm this theory well.

Figure 5 shows the contour charts of the eigenfunction at different wave vectors within the same energy band. The results in (a), (b), (c), and (d) are very simliar to that of the distribution of the electric field. This feature is also consistent with the theory of the energy band in solid state physics. (a)

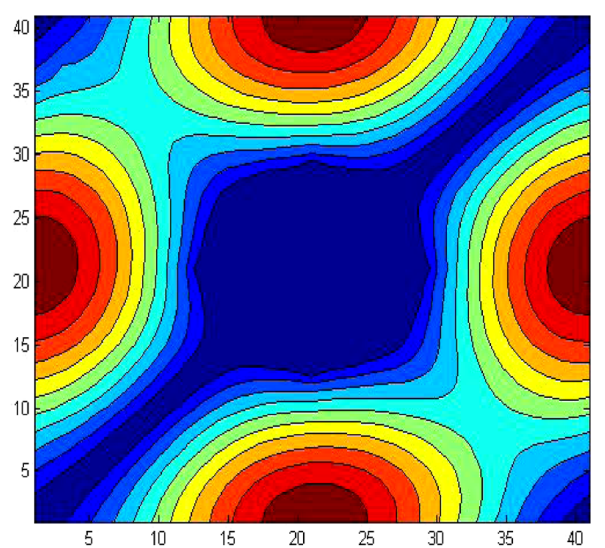

(c)

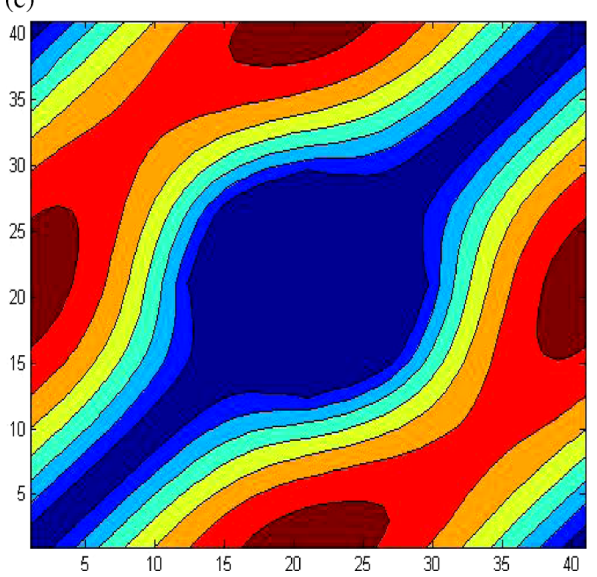

(b)

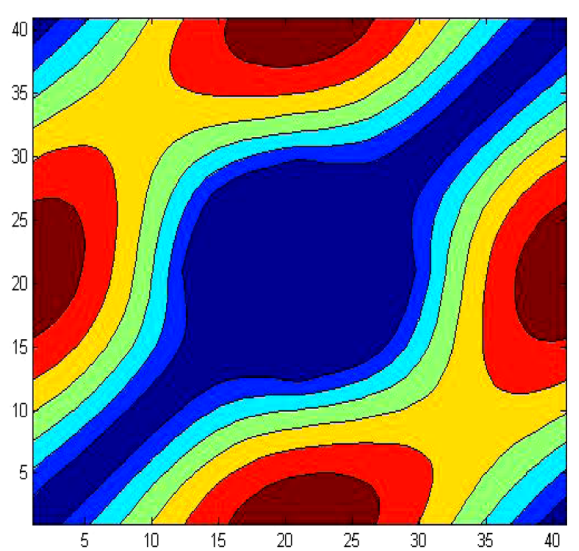

(d)

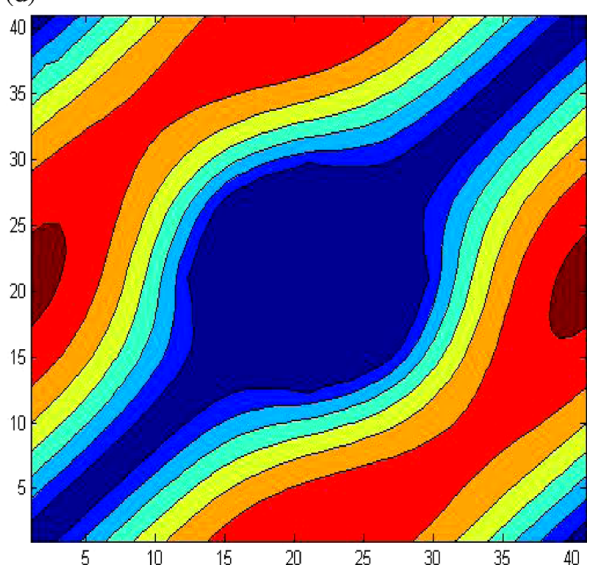

Fig. 5 (colour online). Distribution of the electric field of the eigenmode at different wave vectors within the same energy band (at the second energy band). (a) $k_{x}=k_{y}=0.1$; (b) $k_{x}=k_{y}=0.25$; (c) $k_{x}=k_{y}=0.50$; (d) $k_{x}=k_{y}=0.75$. 


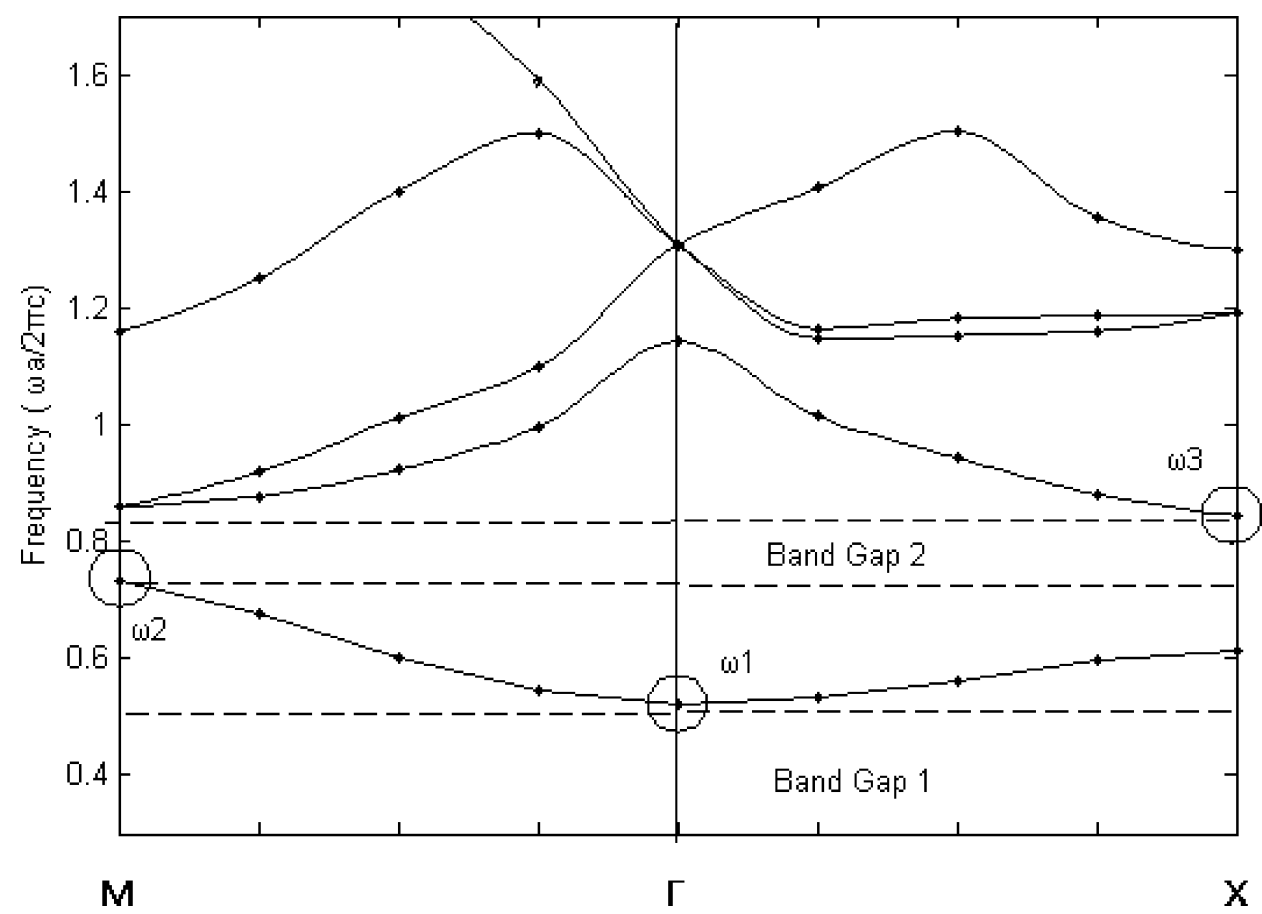

Fig. 6. Electronmagnetic band structure. The eigenfrequencies $\omega_{2}$ and $\omega_{3}$, marked by circles, determine the low- and upperfrequency edges of band gap 2, and for band gap 1, the low-frequency is 0 , the upper-frequency is $\omega_{1}$.

It has been seen from the above analysis that the distribution law of the eigenfunctions can be easily obtained, that is, the eigenfunctions vary widely with the same wave vector and slowly with the same energy band. Thus we can determine the band structure accurately.

Figure 6 shows the electronmagnetic band structure and demonstrats the effectiveness of this approach to determining it. It is found from the calculated results that the 2D metallic photonic crystals have two band gaps for TM polarization. The eigenfrequencies of $\omega_{2}$ and $\omega_{3}$ at the high symmetry points $M$ and $X$ decide the low- and upper-frequency edges of the band gap 2 . Their frequencies are 0.7314 and 0.8548 . The band gap 1 is unique compared with other nonlinear photonic crystals. The lower frequency is 0 and the upper frequency 0.5227 .

[1] E. Yablonovitch, Phys. Rev. Lett. 58, 2061 (1987).

[2] S. John, Phys. Rev. Lett. 58, 2486 (1987).

[3] H. Xiao and D. Z. Yao, Physica E 27, 1 (2005).

[4] D. Wu, Appl. Phys. Lett. 83, 201 (2003).

[5] A. Bingham, Appl. Phys. Lett. 87, 051101 (2005).

[6] F. Raineri, Y. Dumeige, A. Levenson, and X. Letartre, Electron. Lett. 38, 1704 (2002).

\section{Conclusion}

In this letters, the band structure of a $2 \mathrm{D}$ metallic photonic crystal structure with TM polarization has been studied. By analyzing the spatial distribution of the eigenfunction law, i.e. the eigenfunctions vary widely with the same wave vector and slowly with the same energy band, the method to determine the band structure has been obtained. The simulation results well verify the validity of the method.

\section{Acknowledgements}

This work was financially supported by the National Natural Science Foundation of China (Grant No. J0830310).

[7] H. X. Zheng, Int. J. Infrared Millimeter Waves 25, 855 (2005).

[8] K. Skaoda, Phys. Rev. B 64, 045116 (2001).

[9] K. S. Yee, IEEE Transactions on Antennas and Propagation 14, 302 (1966). 